\title{
X-ray position-sensitive duo-lateral diamond detectors at SOLEIL
}

\author{
Kewin Desjardins, ${ }^{a *}$ Michel Bordessoule ${ }^{a}$ and Michal Pomorski ${ }^{b}$ \\ ${ }^{\text {a }}$ Synchrotron SOLEIL, Saint-Aubin, 91192 Gif-sur-Yvette, France, and ${ }^{\mathbf{b}}$ CEA-LIST, Diamond Sensors Laboratory, \\ 91191 Gif-sur-Yvette, France. *Correspondence e-mail: kewin.desjardins@synchrotron-soleil.fr
}

The performance of a diamond X-ray beam position monitor is reported. This detector consists of an ionization solid-state chamber based on a thin singlecrystal chemical-vapour-deposition diamond with position-sensitive resistive electrodes in a duo-lateral configuration. The detector's linearity, homogeneity and responsivity were studied on beamlines at Synchrotron SOLEIL with various beam sizes, intensities and energies. These measurements demonstrate the large and homogeneous (absorption variation of less than $0.7 \%$ over $500 \mu \mathrm{m}$ $\times 500 \mu \mathrm{m})$ active area of the detector, with linear responses independent of the $\mathrm{X}$-ray beam spatial distribution. Due to the excellent charge collection efficiency (approaching $100 \%$ ) and intensity sensitivity $(0.05 \%)$, the detector allows monitoring of the incident beam flux precisely. In addition, the in-beam position resolution was compared with a theoretical analysis providing an estimation of the detector's beam position resolution capability depending on the experimental conditions (X-ray flux, energy and readout acquisition time).

Keywords: X-ray beam position monitor;

XBPM; PSD; diamond detector; noise analysis.

\section{Introduction}

Currently, X-ray beam position monitors are an essential element for synchrotron beamlines that focus the beam down to a few micrometres or less, like the long beamlines using a nano-size X-ray beam for large two-dimensional scan sample mapping (Somogyi et al., 2015). In order to precisely monitor the intensity and adjust the position of the X-ray beams, such beamlines need a fast X-ray diagnostic inserted permanently into the beam (with low absorption and radiation hardness). Many different kinds of in-beam transparent position monitors have been used in the past: microwire devices (Heald, 1986), gaseous detectors (Schildkamp \& Pradervand, 1995), indirect fluorescent or scattered X-ray detection from thin foils with PIN-diodes (Alkire et al., 2000) or pixelated detectors (Rico-Alvarez et al., 2014), as well as thin solid-state devices developed on silicon (Fuchs et al., 2007) or polycrystalline diamond (Bergonzo et al., 1999). In this paper, a new type of diamond X-ray beam position monitor (XBPM) is presented: the device is based on an electronic-grade singlecrystal chemical-vapour-deposition (CVD) diamond material

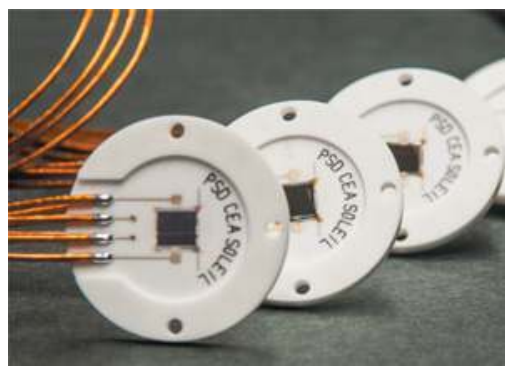
(Morse et al., 2010) processed on a duo-lateral positionsensitive detector (PSD) with diamond-like carbon (DLC) resistive coating (Pomorski et al., 2009). In addition to the excellent physical properties of diamond (radiation hardness, low X-ray absorption, mechanical resistance, large band gap, fast speed charge propagation, etc.), this detector also has several other advantages compared with the classical quadrant XBPM design (Desjardins et al., 2013) or the strip detector (Shu et al., 1998, Zhou et al., 2015): a very large active surface (usable with X-ray beams from millimetre to sub-micrometre 
sizes), easier to calibrate, and a simplified fabrication process. i.e. it does not need any photolithography or precise coating processes. Furthermore, the PSD is an all-carbon device; therefore it represents a good solution for low-energy applications (Desjardins et al., 2014; Gaowei et al., 2015). However, the possible disadvantages, with respect to the quadrant detector, come from the PSD resistive coating, which generates an additional noise source and makes it slower due to the higher RC constant. In this paper, the fabrication of a PSD diamond detector is described, the advantages are presented with experimental results, and the intensity and position monitoring performances are demonstrated.

\section{PSD duo-lateral design}

The PSDs were made from high-quality [100]-oriented electronic-grade single-crystal CVD diamonds from Element Six Ltd (http://www.e6.com) (freestanding windows, type IIae with $\sim 1$ p.p.m. nitrogen, $4.50 \mathrm{~mm} \times 4.50 \mathrm{~mm}$ ) with a thickness of $50 \mu \mathrm{m}$ down to $20 \mu \mathrm{m}$ supplied by ALMAX easyLab (http://www.almax-easylab.com). All plates were previously controlled by cross-polarized microscopy (Hoa et al., 2014) to check that there are no dislocations or other structural defects in the active area frequently rendering diamond devices unstable (Muller et al., 2009). The thickness was measured at SOLEIL by Fourier-transform infrared spectroscopy techniques with a Raman spectroscopy microscope on the SMIS beamline (Dumas et al., 2006) in order to check the homogeneity and parallelism. Additionally, the diamond thickness measurement provides information on the X-ray beam absorption for each detector (Fig. 1). After hot-acid cleaning, both sides of the plates were sputtering-coated with DLC using a simple mask with an opening window of $4 \mathrm{~mm} \times 4 \mathrm{~mm}$, eliminating the need for photolithography. This carbon coating constitutes a resistive layer with the impedance controlled by the deposition time, approximately $100 \mathrm{k} \Omega$ with a thickness of $\sim 200 \mathrm{~nm}$. The two pairs of collecting electrodes at the extremities of both sides were made by sputtering of $\mathrm{Ti} / \mathrm{Au}(\sim 200 \mathrm{~nm})$ layers using a shadow mask (lateral size of $4 \mathrm{~mm} \times 0.25 \mathrm{~mm}$ by electrode). These two electrodes pairs are mutually perpendicularly positioned and the distance between the electrodes of each pair is $3.5 \mathrm{~mm}$.

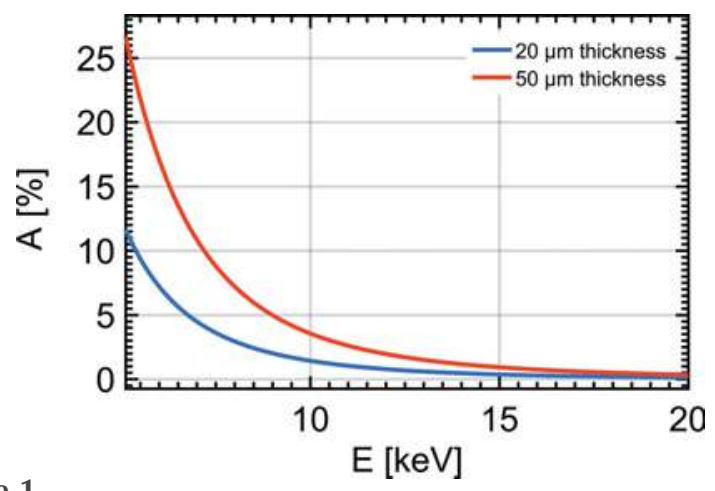

Figure 1

Theoretical X-ray absorption of diamond with thicknesses of $50 \mu \mathrm{m}$ (red curve) and $20 \mu \mathrm{m}$ (blue curve).
The free charge carriers (electron-hole pairs) generated by the X-ray beam interaction in the diamond drift under the influence of the applied electric field created by the externally biased surface electrodes, thus generating an electric current in the external circuit (Fig. 2). In the DLC resistive layer, these charges are divided between the two electrodes on both surfaces; therefore the X-ray beam position (as defined by the centre of gravity of the beam) is directly obtained from the corresponding currents and the PSD dimensions as written in equation (1),

$$
X=K_{x} \frac{I_{x 1}-I_{x 2}}{I_{x 1}+I_{x 2}}, \quad Y=K_{y} \frac{I_{y 1}-I_{y 2}}{I_{y 1}+I_{y 2}}
$$

where $x$ and $y$ are coordinates of the position of the X-ray beam center, $K_{x}$ and $K_{y}$ are the scale factors equal to $L / 2$ with $L$ the distance between collecting electrodes, and $I\left(x_{1,2}\right.$ or $\left.y_{1,2}\right)$ is the signal collected from the horizontal $(x)$ and vertical $(y)$ electrode pairs, respectively.

The total intensity is given by the sum of the signal collected from one of the electrode pairs (i.e. $I_{\mathrm{t}}=\left|I_{x 1}+I_{x 2}\right|=\left|I_{y 1}+I_{y 2}\right|$ ) and is directly proportional to the part of the absorbed X-ray beam [assuming a total charge collection efficiency equal to $100 \%$. It can be estimated from equation (2),

$$
I_{\mathrm{t}}=q \frac{E_{\mathrm{ph}}}{\varepsilon_{\mathrm{p}}} A\left(E_{\mathrm{ph}}\right) \Phi
$$

where $I_{\mathrm{t}}$ is the total current produced by the XBPM, $q$ is the value of the electron charge, $E_{\mathrm{ph}}$ is the incident-beam energy, $\varepsilon_{\mathrm{p}}=13.25 \pm 0.5 \mathrm{eV}$ is the electron-hole pair creation energy (Keister \& Smedley, 2009) for X-ray absorption in diamond, $A$ is the absorption factor depending on the energy $E_{\mathrm{ph}}$ and diamond thickness, and $\Phi$ is the X-ray beam flux.

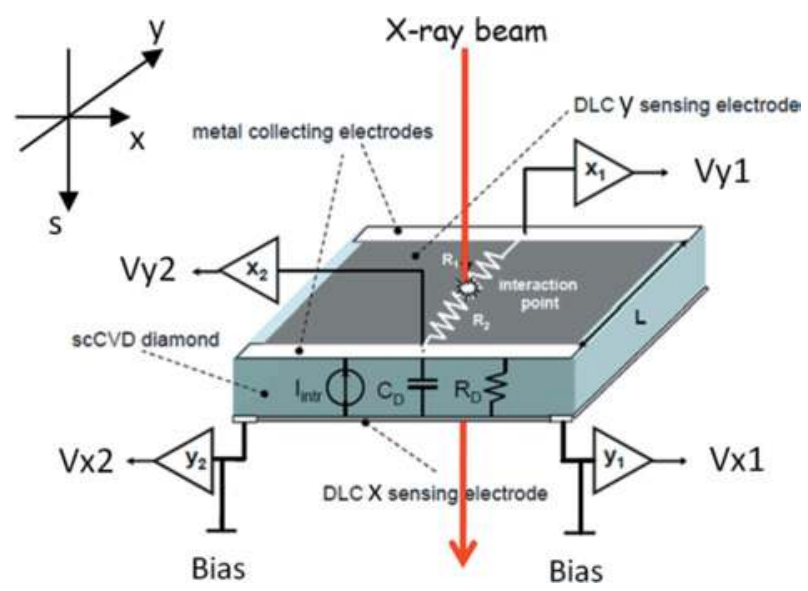

Figure 2

Schematic diagram of the duo-lateral PSD. Inter-electrode area, $L \times L$, with orthogonal opposed electrode on the front and back sides. Four current-to-voltage amplifier channels with bias applied to the back side. $C_{\mathrm{D}}, R_{\mathrm{D}}$ and $I_{\text {intr }}$ are, respectively, the capacitance, the impedance and the leakage current of the single-crystal CVD diamond. 

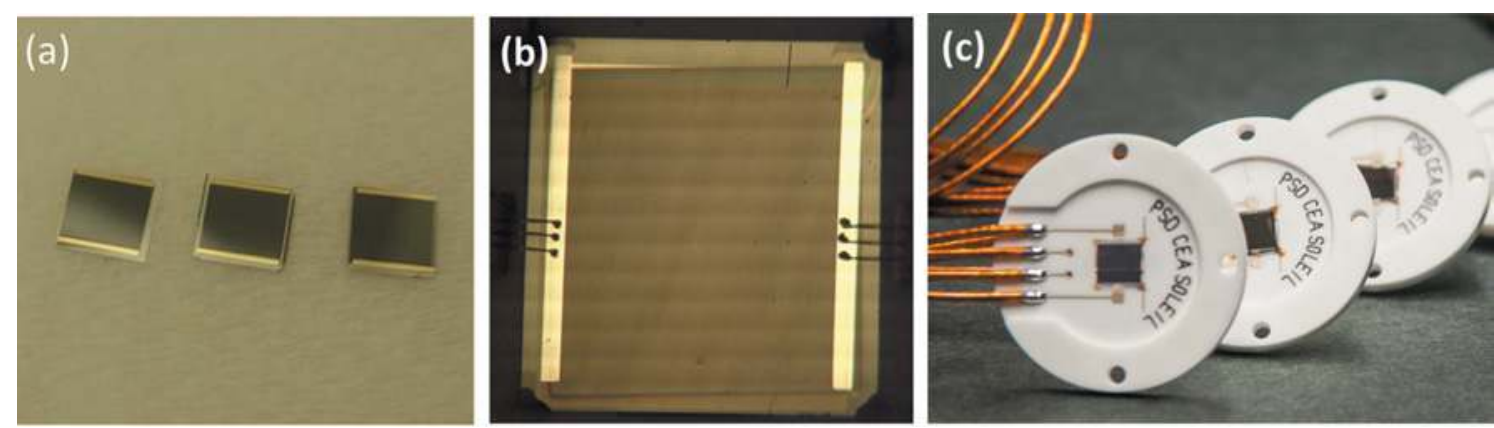

Figure 3

(a) XBPM PSDs. (b) Active area with DLC and two electrodes wire-bounded. (c) XBPM PSD series (50 $\mu \mathrm{m}$ down to $20 \mu \mathrm{m}$ thickness) mounted on boards.

\section{PSD diamond results}

\subsection{Experimental set-up and electronics}

Several PSDs (Fig. 3a) were mounted on specific ceramic boards where the electrodes were connected with aluminium wedge wire bondings (Figs. 3b, 3c). One of these XBPMs was mounted on the GALAXIES beamline (Céolin et al., 2013) and tested at an energy of $6.2 \mathrm{keV}$ with a high photon flux of $1.7 \times 10^{13}$ photons s$^{-1}$ and a small beam spot of $30 \mu \mathrm{m} \times$ $90 \mu \mathrm{m}$. Two other PSDs have been installed on the METROLOGIE (Idir et al., 2006) and SIRIUS beamlines (Ciatto et al., 2016) for spatial resolution measurements with beam energies of $7 \mathrm{keV}$ and $2.6 \mathrm{keV}$, respectively, and photon fluxes down to $10^{10}$ photons s ${ }^{-1}$. Finally, a micro beam position reconstruction capability has been demonstrated with an $\mathrm{X}$-ray beam focused by a zone plate $(1 \mu \mathrm{m} \times 1 \mu \mathrm{m})$ on the NANOSCOPIUM beamline (Somogyi et al., 2015) with a beam energy of $12 \mathrm{keV}$ and a flux of $10^{10}$ photons s ${ }^{-1}$.

Each pair of PSD electrodes $(X$ and $Y$ ) were connected to the current-to-voltage amplifier (IV-amp) LOCUM-4F (ENZ, http://www.enz-de.de). This IV-amp has four channels for measuring simultaneously the four low currents (ranging from $1 \mathrm{~mA}$ down to $100 \mathrm{pA}$ ), and it is particularly adapted for PSD use with a splitter bias, which is able to apply a voltage only to one side of the PSD ( $X$ electrodes in Fig. 2), while the two other channels remain connected to the main ground $(0 \mathrm{~V})$ potential ( $Y$ electrodes in Fig. 2). However, the front-end circuit of the LOCUM is not specified in terms of noise. Finally, the IV-amp output voltages were digitized by an analog-to-digital convertor (ADC Adlink 2005, http://www. adlinktech.com) and recorded by TANGO control applications (http://www.tango-controls.org)

\subsection{Detector calibration}

The PSD position calibration factors (or scale factors) were determined experimentally by translating the detector linearly in the X-ray beam, recording the four currents (Fig. 4a) and plotting values of difference/sum currents versus the position (Fig. $4 b$ ). The results were fit by equation (1) in the central region for both directions to give the two scale factors $\left(K_{x}\right.$ and $\left.K_{y}\right)$. This experimental result assures excellent position reconstruction and compensation for fabrication defects or angle misalignment. In comparision with the classical diamond quadrant XBPM, these calibration factors are stable and do not depend on the spatial X-ray beam distribution. A comparison between the vertical calibration factors obtained for the different beam sizes (as defined by slits) with the PSD device (green curve) and quadrant detector (blue line) for the same in-beam conditions is shown in Fig. 5. An additional measurement has also been performed with a focused X-ray

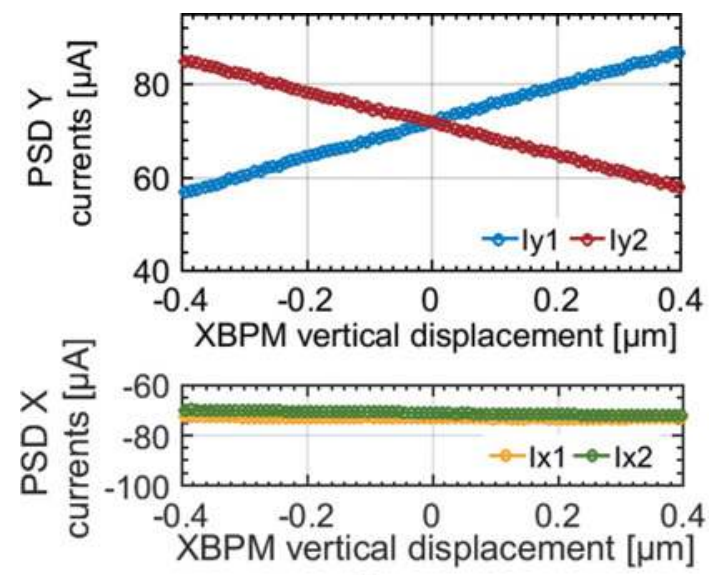

(a)

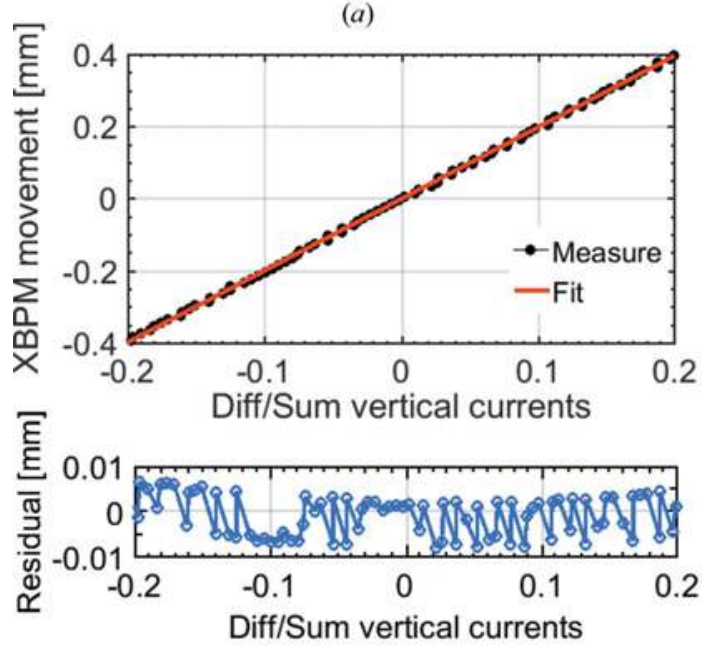

(b)

Figure 4

(a) Measured PSD currents versus XBPM displacement through the beam in the vertical direction: vertical currents (top) and horizontal currents (bottom). (b) Measured PSD difference over the sum currents versus XBPM displacement in the vertical direction and linear fit residual. 


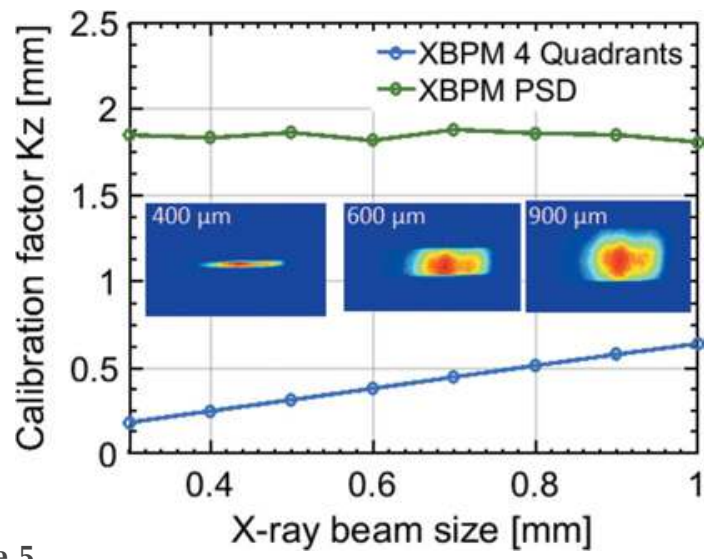

Figure 5

Comparison between the calibration factors obtained with the quadrant XBPM (blue line) and the PSD XBPM (green line) for different X-ray beam sizes. The scale factor of the quadrant XBPM depends linearly on beam size. The scale factor of the PSD is stable.

beam $(1 \mu \mathrm{m} \times 1 \mu \mathrm{m})$ on the NANOSCOPIUM beamline (Fig. 6). It allows demonstration of the beam position reconstruction with very small beam over a large area $(1 \mathrm{~mm})$ which is not a simple task with a classical quadrant detector (Bergonzo et al., 1999).

Therefore, the reconstructed position could be measured independently of the experimental conditions (energy, slit gap, focused or unfocused beam, etc.).

\subsection{Flux and intensity monitoring capabilities}

The total charge collection efficiency was verified on the METROLOGIE beamline by measuring the X-ray beaminduced current (XBIC) as a function of the bias voltage, as shown in Fig. 7. Like the other diamond XBPMs made with electronic-grade single-crystal CVD (Desjardins et al., 2013), a potential of a few Volts $\left(>0.2 \mathrm{~V} \mathrm{\mu m}^{-1}\right)$ is enough to reach constant XBIC values. Thereby, the flux can be easily calculated as equation (2).

The dark current depends on the PSD inter-electrode resistance $\left(R_{\mathrm{ie}}\right)$, the temperature and the input voltage offsets of the operational amplifier. Its intensity is rather high with low resistance but significantly lower $(<1 \mathrm{nA})$ with $R_{\mathrm{ie}}>$

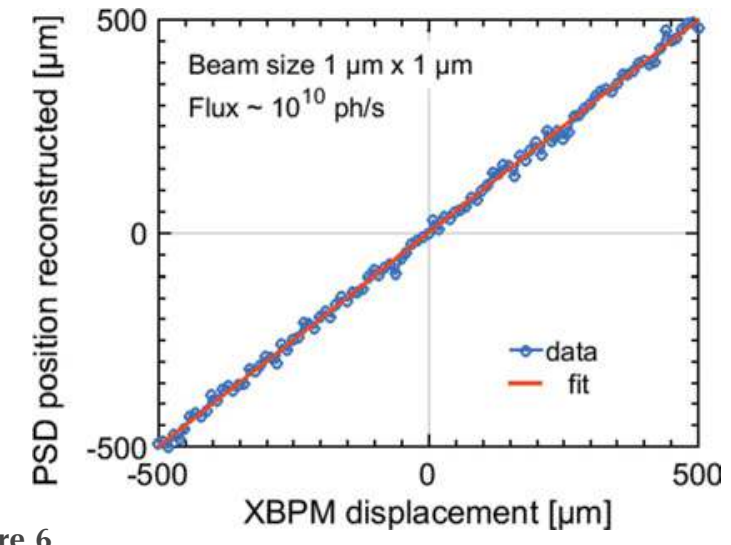

Figure 6

The PSD reconstructed position of a linear movement over $1 \mathrm{~mm}$ with a $1 \mu \mathrm{m} \mathrm{X}$-ray beam $\left(\right.$ flux $\simeq 10^{10}$ photons $\mathrm{s}^{-1}$ ).

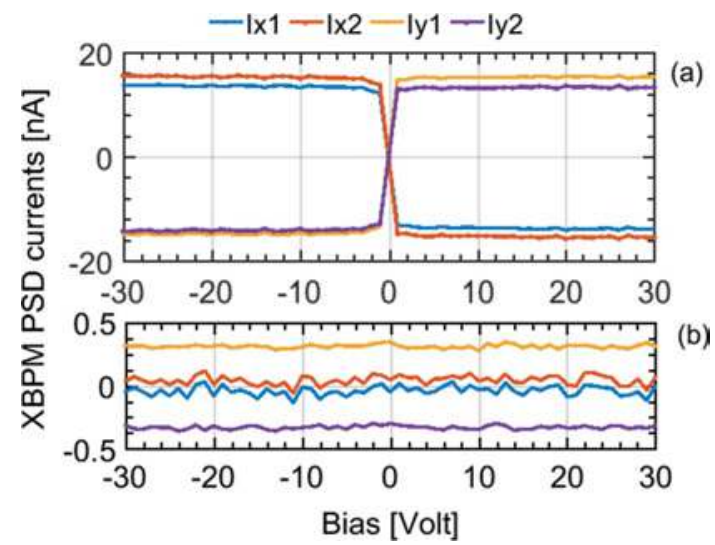

Figure 7

Top: PSD X-ray beam-induced current (XBIC) versus detector bias. Bottom: PSD dark current versus detector bias.

$100 \mathrm{k} \Omega$. However, the stable dark currents do not influence the position measurement precision, due to the easily applicable corrections. On the other hand, at low X-ray fluxes, the photocurrent reaches offset levels, thereby defining a reasonable usability limit for this detector. Furthermore, these dark currents may be subtracted from each current for efficient estimation of the incident X-ray photon flux.

The XBPM intensity monitoring capability has been evaluated on the GALAXIES beamline, and the obtained sensitivity is illustrated in Fig. 8 with a low XBIC fluctuation. The perfect visualization of the top-up filling mode of the storage ring at SOLEIL (with $0.5 \%$ electron re-injection every few minutes) can be seen, which is in excellent agreement with silicon diode measurements (error $<0.05 \%$ ).

The homogeneity is determined on the METROLOGIE beamline (flux $\simeq 10^{10}$ photons $^{-1}$ ) from a two-dimensional scan which is plotted in Fig. 9 with an observed signal variation below $0.7 \%$ over $500 \mu \mathrm{m} \times 500 \mu \mathrm{m}$ of active area. This is clearly attributable to the thickness non-uniformity of the diamond plate corresponding here to a peak-to-peak thickness difference of $300 \mathrm{~nm}$ at an X-ray energy of $7 \mathrm{keV}$.

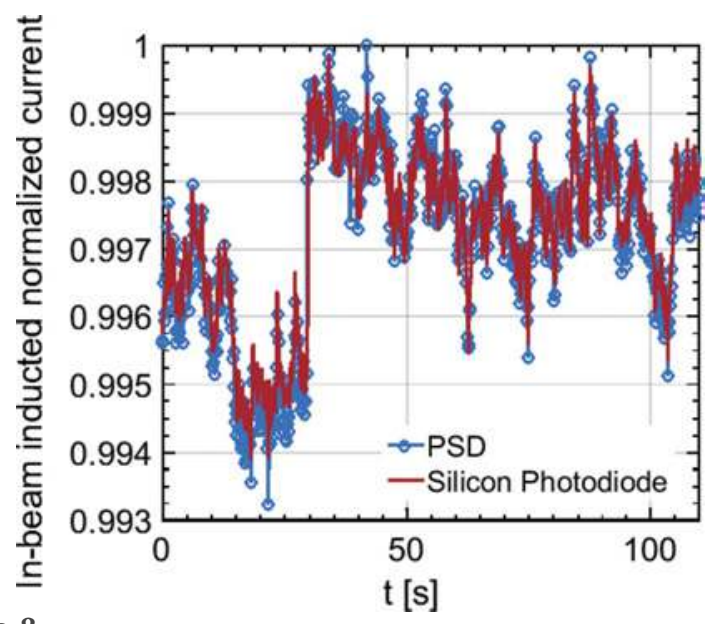

Figure 8

Time scan showing the top-up mode of operation of the SOLEIL storage ring with a re-injection at $t=30 \mathrm{~s}$. XBPM PSD diamond (blue) and the corresponding downstream Si photodiode (red) current signals. 


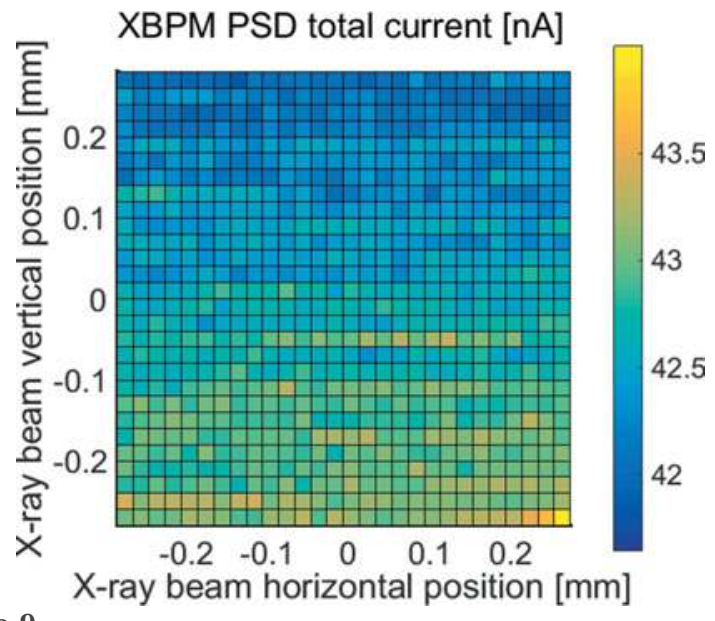

Figure 9

Total current, $I_{\mathrm{t}}$, map made over the central area $(500 \mu \mathrm{m} \times 500 \mu \mathrm{m})$ of the PSD (X-ray energy of $7 \mathrm{keV}$ and flux $\simeq 10^{10}$ photons $\mathrm{s}^{-1}$ ).

Hence, the intensity and flux can be easily measured independently of the X-ray beam characteristics (energy, spatial distribution, focused or unfocused beam, etc.).

\subsection{Beam position monitoring capabilities}

A first and simple demonstration of the PSD position measurement capability consisted of the reconstruction of the position for small PSD displacements with a high beam flux $\left(>10^{13}\right.$ photons s $^{-1}$ ) on the GALAXIES beamline (Fig. 10). Beam displacements of $5 \mu \mathrm{m}, 1 \mu \mathrm{m}, 500 \mathrm{~nm}$ and $100 \mathrm{~nm}$ could be reconstructed under these conditions (ADC integration time of $100 \mathrm{~ms}$, LOCUM-4F bandwidth $=2.5 \mathrm{kHz}$, total $\mathrm{XBIC}=140 \mu \mathrm{A}$ ) with a standard deviation of $50 \mathrm{~nm}$ r.m.s. (when the beam is assumed to be stable).

To understand the noise contributions associated with a beam position measurement, a model is proposed where the instrumental noise is distinguished from the photonic noise and X-ray beam noise. Also, this model is compared with the measurement from a large range of $X$-ray beam currents.

In equation (1), the position $P$ on an axis $(X$ or $Y)$ is defined by the ratio of the difference over the sum of currents. In

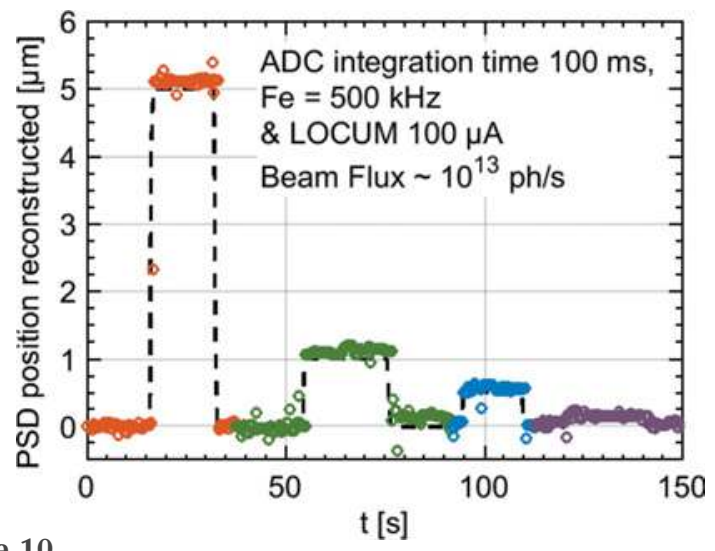

Figure 10

Diamond PSD reconstructed position for very small displacements: $5 \mu \mathrm{m}, 1 \mu \mathrm{m}, 0.5 \mu \mathrm{m}$ and $0.1 \mu \mathrm{m}$ (X-ray energy of $6.2 \mathrm{keV}$ and flux $\simeq$ $10^{13}$ photons s$^{-1}$ ).
Table 1

Sources of noise for each PSD current and their correlation.

\begin{tabular}{|c|c|}
\hline $\begin{array}{l}\text { Independent } \\
\text { noise }\end{array}$ & $\begin{array}{l}\sigma_{001}^{2}, \sigma_{012}^{2} \text { are composed of the low amplifier self-noise, } \\
\sigma_{0101}^{2}, \text { and the ADC noise, } \sigma_{3 d .}^{2} .\end{array}$ \\
\hline $\begin{array}{l}\text { Correlated } \\
\text { noise }\end{array}$ & $\begin{array}{l}\sigma_{\mathrm{co}}^{2} \text { results from the X-ray beam flux noise (unmodelled), } \\
\text { the eddy currents (unmodelled) and the shot noise } \sigma_{\mathrm{id}}^{2} \\
\text { and } \sigma_{\mathrm{ic}}^{2} \text { of the difference and sum currents, respectively. }\end{array}$ \\
\hline $\begin{array}{l}\text { Anti-correlated } \\
\text { noise }\end{array}$ & $\begin{array}{l}\sigma_{\mathrm{ac}}^{2} \text { is a combination of the unmodelled noise from the } \\
\text { X-ray beam vibration }\left(\sigma_{\mathrm{bv}}^{2}\right) \text { and the dominant noise } \\
\left(\sigma_{\mathrm{iRie}}^{2}\right) \text { caused by the resistive }\left(R_{\mathrm{ie}}\right) \text { coupling of the } \\
\text { IV-amp's inputs. This noise has not been analyzed } \\
\text { here (Van Esch et al. } 2004) \text {. }\end{array}$ \\
\hline
\end{tabular}

equation (3), we denote $D$ for the differential current $(I 1-I 2)$ and $C$ for the common-mode current $(I 1+I 2)$ crossing the detector; the scale factor is now denoted $K$,

$$
P=K \frac{D}{C} \text {. }
$$

In the following text, $\mu$ refers to a mean and $\sigma$ to a standard deviation. For instance, the position $P$ has a mean, $\mu_{\mathrm{p}}$, and a variance, $\sigma_{\mathrm{p}}^{2}$, given in equation (4). The latter derives from the variance of the ratio $D / C$ (Seltman, 2017). It is further shown that the covariance, $\sigma_{\mathrm{dc}}^{2}$, is small,

$$
\sigma_{\mathrm{p}}^{2}=\frac{\mu_{\mathrm{d}}^{2}}{\mu_{\mathrm{c}}^{2}}\left[\frac{\sigma_{\mathrm{d}}^{2}}{\mu_{\mathrm{d}}^{2}}+\frac{\sigma_{\mathrm{c}}^{2}}{\mu_{\mathrm{c}}^{2}}-2 \frac{\sigma_{\mathrm{dc}}^{2}}{\mu_{\mathrm{d}} \mu_{\mathrm{c}}}\right] .
$$

$\sigma_{\mathrm{d}}^{2}$ and $\sigma_{\mathrm{c}}^{2}$ are, respectively, the difference and the sum noises and are given in equations (7) and (9); $\sigma_{\mathrm{dc}}^{2}$ is the covariance between $D$ and $C$.

Also, each PSD current has a noise, which is the sum of the correlated and independent noise, and is written as

$$
\sigma_{I 1}^{2}=\sigma_{0 I 1}^{2}+\sigma_{\mathrm{co}}^{2}+\sigma_{\mathrm{ac}}^{2}, \quad \sigma_{I 2}^{2}=\sigma_{0 I 2}^{2}+\sigma_{\mathrm{co}}^{2}+\sigma_{\mathrm{ac}}^{2},
$$

where the different types of noise are described in Table 1.

In practice, each electronic channel is similar $\left(\sigma_{0 I 1}^{2}=\sigma_{0 I 2}^{2}=\right.$ $\left.\sigma_{0}^{2}\right)$ and the associated electronic noise is very low $\left(<1 \mathrm{pA} \mathrm{Hz}^{-1 / 2}\right)$ compared with the other noise sources, which implies that the covariance $\left(\sigma_{\mathrm{dc}}^{2}=\sigma_{0 I 1}^{2}-\sigma_{0 I 2}^{2}\right)$ is negligible. Furthermore, if the beam is centred $\left(\mu_{\mathrm{p}}=0\right)$, the term $\sigma_{\mathrm{c}}^{2}$ in equation (4) vanishes, and the expression of the position noise reduces to equation (6),

$$
\sigma_{\mathrm{p}}=K \frac{\sigma_{\mathrm{d}}}{\mu_{\mathrm{c}}}
$$

Hence, the centred position noise is proportional to the differential noise, and inversely proportional to the detector current. The differential noise, $\sigma_{\mathrm{d}}^{2}$, is modelled according to equation (7),

$$
\sigma_{\mathrm{d}}^{2}=\left[\sigma_{\mathrm{id}}^{2}+4 \sigma_{\mathrm{ac}}^{2}\right] H 1(f)^{2}+\sigma_{\mathrm{adc}}^{2},
$$

where $\sigma_{\mathrm{id}}^{2}$ is the current noise, $\sigma_{\mathrm{ac}}^{2}$ is the anti-correlated noise (Table 1 ), $\sigma_{\mathrm{adc}}^{2}=11 \mathrm{fA} \mathrm{Hz}^{-1 / 2}$ is the ADC quantization noise, which appears to be partially correlated, likely due to some clock jitter, and $H 1(f)$ is the module of the trans-impedance amplifier frequency response [equation (8)],

$$
H 1(f)=\left\{\left[1+(f / F 1)^{2}\right]\left[1+(f / F 2)^{2}\right]\right\}^{-1 / 2} .
$$


Table 2

Detector characteristics, beamline experimental conditions, acquisition characteristics and the parameters of the noise model used.

\begin{tabular}{|c|c|}
\hline PSD & $\begin{array}{l}\text { Diamond thickness } 26 \mu \mathrm{m}, R_{\mathrm{ie}}(X)=25 \mathrm{k} \Omega \text { and } \\
\qquad R_{\mathrm{ie}}(Y)=45 \mathrm{k} \Omega\end{array}$ \\
\hline X-ray beam & $\begin{array}{l}\text { METROLOGIE beamline (bending magnet), } \\
10^{8} \text { photons s }{ }^{-1} \text { to } 10^{10} \text { photons s }{ }^{-1} \text { and } E_{\mathrm{ph}}=7 \mathrm{keV}\end{array}$ \\
\hline IV-amp & $\begin{array}{l}\text { LOCUM- } 4 \mathrm{~F} \text { with } I_{\text {range }}=100 \mathrm{nA}[\text { i.e. } \mathrm{RF}=100 \mathrm{M} \Omega \\
\left.\text { output }=1 \mathrm{~V} \mathrm{nA}^{-1} \text { and } \mathrm{BP}(3 \mathrm{~dB})=360 \mathrm{~Hz}\right]\end{array}$ \\
\hline $\begin{array}{l}\text { IV-amp filter } \\
\text { parameters } \\
\text { [equation (8)] }\end{array}$ & $F 1=230 \mathrm{~Hz}$ and $F 2=2800 \mathrm{~Hz}$ \\
\hline ADC & $\begin{array}{l}\text { ADC Adlink } 2005 \text { with sampling frequency } \\
F_{\mathrm{s}}=200 \mathrm{kHz}\left(F_{\mathrm{Nyq}}=100 \mathrm{kHz}\right), 16 \text { bit with noise of } \\
2 \text { bit }(\text { dynamic } 14 \mathrm{bit})\end{array}$ \\
\hline $\begin{array}{l}\text { ADC noise } \\
\text { Anti-correlated } \\
\text { noise model } \\
\text { fitted }\end{array}$ & $\begin{array}{l}\sigma_{\text {adc }}=I_{\text {Range }} /\left[2^{14}\left(12 F_{\mathrm{Nyq}}\right)^{1 / 2}\right]=10 \mathrm{fA} \mathrm{Hz}^{-1 / 2} \\
\sigma_{\mathrm{ac}}=1.8 \mathrm{pA} \mathrm{Hz} \mathrm{Hz}^{-1 / 2}+\left(10 \mathrm{pA} \mathrm{Hz} f^{-1 / 2}\right)\end{array}$ \\
\hline
\end{tabular}

As we wanted to consider the sum noise $\left(\sigma_{\mathrm{c}}^{2}\right)$ in this study, we give it in equation (9),

$$
\sigma_{\mathrm{c}}^{2}=\left[\sigma_{\mathrm{ic}}^{2}\right] H 1(f)^{2}+2 \sigma_{\mathrm{adc}}^{2} .
$$

The shot noise (Spear, 2005) difference, $\sigma_{\text {id }}^{2}$, and sum, $\sigma_{\text {ic }}^{2}$, remain to be defined in equations (10) and (11) to complete this noise model,

$$
\begin{gathered}
\sigma_{\mathrm{id}}^{2}=2 q \mu_{\mathrm{d}}\left(\mathrm{FF}+E_{\mathrm{ph}} / \varepsilon_{\mathrm{p}}\right), \\
\sigma_{\mathrm{ic}}^{2}=2 q \mu_{\mathrm{c}}\left(\mathrm{FF}+E_{\mathrm{ph}} / \varepsilon_{\mathrm{p}}\right),
\end{gathered}
$$

where $q$ is the value of the electron charge, $E_{\mathrm{ph}}$ is the energy of the incident beam, $\varepsilon_{\mathrm{p}}=13.25 \pm 0.5 \mathrm{eV}$ is the electron-hole pair creation energy for X-ray absorption in diamond, and $\mathrm{FF}=0.08$ is the Fano factor of diamond.

This established model was compared with a series of noise density measurements performed on the METROLOGIE beamline. A diamond PSD was irradiated with different X-ray fluxes $\left(10^{8}\right.$ photons $\mathrm{s}^{-1}$ to $10^{11}$ photons $\left.\mathrm{s}^{-1}\right)$ by insertion of various metallic absorbers into the beam. The ADC sampling frequency was $200 \mathrm{kHz}$ with an integration time of $1 \mathrm{~s}$ which allows the acquisition of 200000 samples per measurement. The LOCUM current range ( $\left.I_{\text {range }}\right)$ was adapted to the XBIC level. At every flux level, varying the corresponding total PSD current between $4 \mathrm{nA}$ and $130 \mathrm{nA}, 20$ spectra corresponding to the fast Fourier transfer of the ADC data were successively acquired. Table 2 summarizes the detector characteristics, the experimental conditions and the parameters of the noise model used.

Figs. 11( $a$ ) and 11(b) give examples of two acquired spectra for a total photocurrent of $\mu_{\mathrm{c}}=88.1 \mathrm{nA}$ for the sum and difference, respectively, in comparison with the models given by equations (7) and (9).

Excess noise appears in the sum noise (Fig. 11a) as peaks $(50 \mathrm{~Hz}$ and $70 \mathrm{~Hz})$. This noise does not come from the amplifier, because its variance is proportional to the total current. This is a flux noise, which will not be converted as position noise [equation (6)]. Similarly, in the difference noise (Fig. 11b), the harmonic frequencies from the local electrical
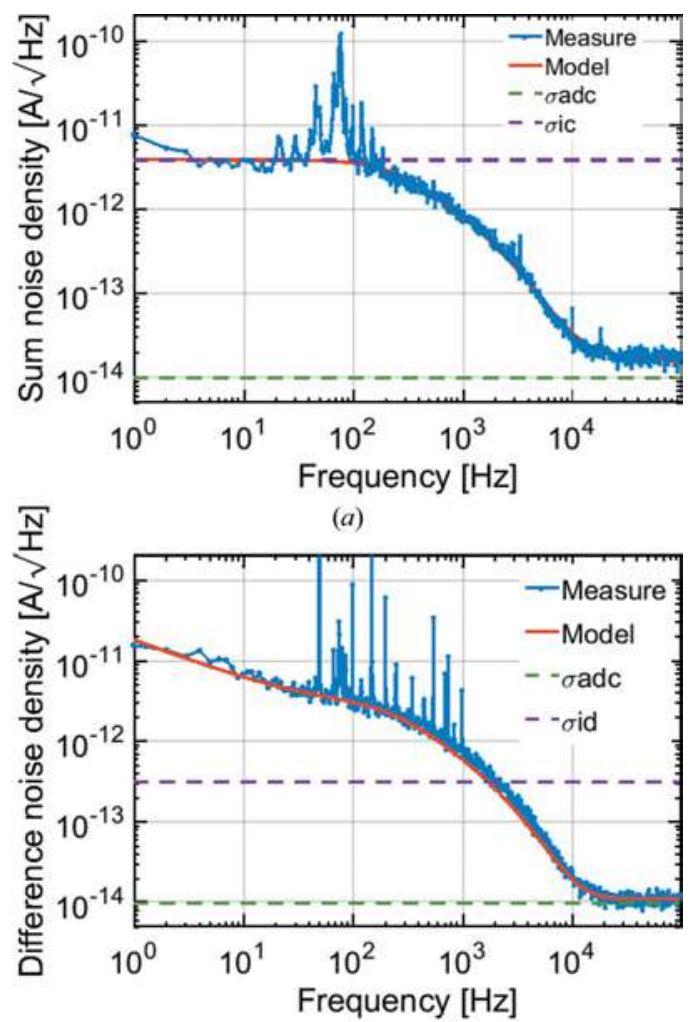

Figure 11

(b)

(a) Measured and model of the sum of the current density spectrum. (b) Measured and model of the difference of the current density spectrum. The blue lines are the measurement, the red lines are the models of $\sigma_{\mathrm{c}}^{2}$ and $\sigma_{\mathrm{d}}^{2}$. The green dashed line is $\sigma_{\mathrm{adc}}^{2}$ and the magenta dashed line is the shot noise, $\sigma_{\mathrm{ic}}^{2}$ and $\sigma_{\mathrm{id}}^{2}$. The total current is $\mu_{\mathrm{c}}=88 \mathrm{nA}$ and $\mu_{\mathrm{d}}=0.6 \mathrm{nA}$.

supply network are present. This defect comes from the amplifier. This noise will be converted as position noise.

With these observations, and knowing that the current difference shot noise $\left(\sigma_{\mathrm{id}}^{2}\right)$ and the beam vibration noise $\left(\sigma_{\mathrm{bv}}^{2}\right)$ are both low, the difference noise is considered constant. The position noise is then calculated from equations (6) and (7) and compared in Fig. 12 for two incident X-ray fluxes. The position noise is always the same shape, with the intensity proportional to the total current, as stated in equation (6).

Finally, it is possible to trace a complete diagram giving the theoretical PSD limit of the position resolution as a function of the X-ray beam-induced current (depending on the diamond thickness, the beam flux and energy used) and the acquisition bandwidth. In Fig. 13 we have compiled the measurement results obtained for a bandwidth of $40 \mathrm{~Hz}$ at the METROLOGIE and SIRUS beamlines obtained with two different PSD devices and compare it with the presented model. The corresponding result obtained at the GALAXIES beamline and presented in Fig. 10 is also included. All of these demonstrate excellent accordance with the theoretical model.

\section{Conclusion}

The feasibility of building a duo-lateral position-sensitive detector based on CVD diamond has been demonstrated in 


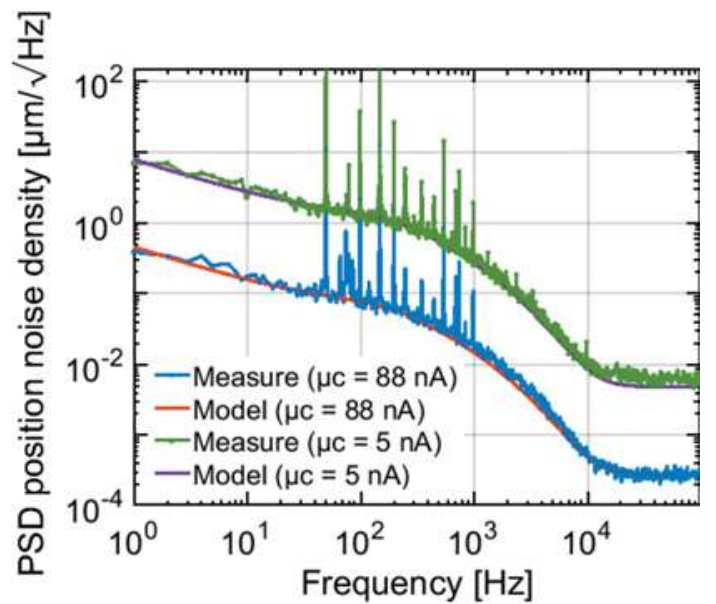

Figure 12

Two measured position noise density spectra compared with their models with total currents of $\mu_{\mathrm{c}}=88.1 \mathrm{nA}$ (blue and red) and $\mu_{\mathrm{c}}=5 \mathrm{nA}$ (green and purple).

this paper. Its excellent capabilities of intensity and flux monitoring on the same level as the more common diamond quadrant XBPMs have been confirmed. Additionally, the various advantages of PSDs have been shown, such as their simpler position calibration, and the large active area for both large and very small beam dimensions. The PSDs are particularly adapted to beamlines where the X-ray beam spatial distribution could be modified during the experiment (i.e. during energy scans and monochromator movements) or for synchrotron beamlines which do not use a position feedback device. The principal drawbacks linked to the noise sources have been discussed and a theoretical model has been compared with measured data, which permit the optimization of the diamond PSD knowing the experimental beamline conditions (i.e. energy, incident flux and especially the beam

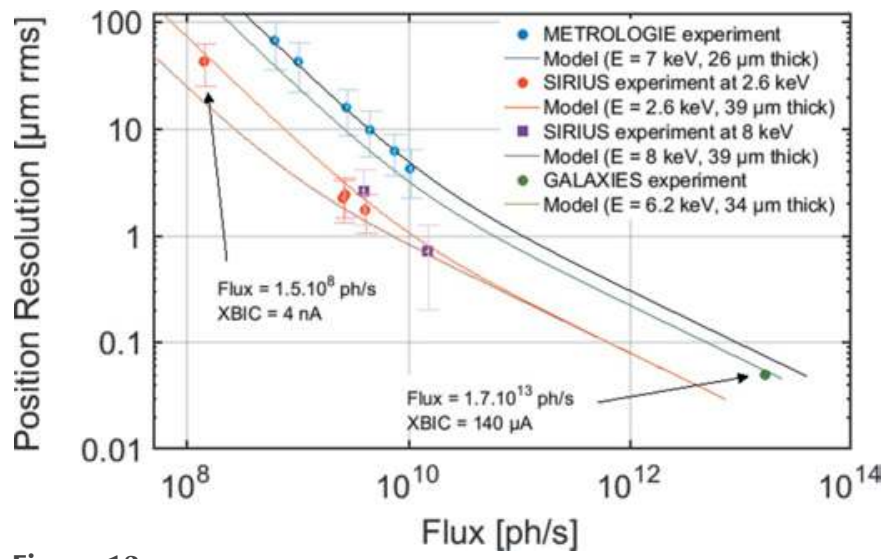

Figure 13

Position resolution measured versus the theoretical model for different PSD and X-ray beam fluxes. The blue, red and violet dots represent the measured PSD resolution (sum of position noise density between $10 \mathrm{~Hz}$ to $40 \mathrm{~Hz}$ ) obtained on the METROLOGIE and SIRIUS beamlines, respectively, for different incident fluxes. The green dot shows a similar measurement obtained on the GALAXIES beamline corresponding to the measurement standard deviation when the beam is stable (Fig. 10). The lines are the calculated theoretical limits of the reconstructed position for these PSDs, the beamline configurations and the acquisition bandwidth. position precision measurement needed in the sample) with a compromise between the expected generated X-ray beam current, the XBPM absorption and desired readout acquisition speed.

Finally, PSD damond XBPMs have been installed on 12 beamlines at Synchrotron SOLEIL and at two other synchrotrons (NSLSII and SSRF). Additionally, a very thin device has been made (CVD diamond of $4 \mu \mathrm{m}$ thickness) for the SIRIUS beamline at SOLEIL for use in the tender X-ray range in order to upgrade the previously installed thin quadrant diamond detector.

\section{Acknowledgements}

We would like to thank the GALAXIES, SIRIUS, NANOSCOPIUM and particularly the METROLOGY beamline teams for their support and beam time necessary for the characterization of the PSD XBPM.

\section{References}

Alkire, R. W., Rosenbaum, G. \& Evans, G. (2000). J. Synchrotron Rad. 7, 61-68.

Bergonzo, P., Brambilla, A., Tromson, D., Marshall, R. D., Jany, C., Foulon, F., Gauthier, C., Solé, V. A., Rogalev, A. \& Goulon, J. (1999). J. Synchrotron Rad. 6, 1-5.

Céolin, D., Ablett, J. M., Prieur, D., Moreno, T., Rueff, J. P., Marchenko, T., Journel, L., Guillemin, R., Pilette, B., Marin, T. \& Simon, M. (2013). J. Electron Spectrosc. Relat. Phenom. B, 190, 188-192.

Ciatto, G., Chu, M. H., Fontaine, P., Aubert, H., Renevier, H. \& Deschanvres, J. L. (2016). Thin Solid Films, 617, 48-54.

Desjardins, K., Duran, D., Hustache, S., Pomorski, M. \& Shepard, W. (2013). J. Phys. Conf. Ser. 425, 212004.

Desjardins, K., Pomorski, M. \& Morse, J. (2014). J. Synchrotron Rad. 21, 1217-1223.

Dumas, P., Polack, F., Lagarde, B., Chubar, O., Giorgetta, J. L. \& Lefrançois, S. (2006). Infrared Phys. Technol. 49, 152-160.

Fuchs, M. R., Holldack, K., Reichardt, G. \& Mueller, U. (2007). AIP Conf. Proc. 879, 1006-1009.

Heald, S. M. (1986). Nucl. Instrum. Methods Phys. Res. A, 246, 411412.

Hoa, L. T. M., Ouisse, T., Chaussende, D., Naamoun, M., Tallaire, A. \& Achard, J. (2014). Cryst. Growth Des. 14, 5761-5766.

Idir, M., Mercère, P., Moreno, T. \& Delmotte, A. (2006). Synchrotron Radiat. News, 19, 18-23.

Keister, J. W. \& Smedley, J. (2009). Nucl. Instrum. Methods Phys. Res. $A$, 606, 774-779.

Gaowei, M., Smedley, J., Zhou, T., Muller, E. \& Sumant, A. (2015). Proceedings of the Sixth International Particle Accelerator Conference (IPAC2015), 3-8 May 2015, Richmond, VA, USA.

Morse, J., Solar, B. \& Graafsma, H. (2010). J. Synchrotron Rad. 17, 456-464.

Muller, E., Smedley, J., Raghothamachar, B., Gaow, M., Keister, J., Ben-Zvi, I., Dudley, M. \& Wu, Q. (2009). MRS Online Proceeding Library Archive 1203.

Pomorski, M., Ciobanu, M., Mer, C., Rebisz-Pomorska, C., Tromson, D. \& Bergonzo, P. (2009). Phys. Status Solidi A, 206, 2109-2114.

Rico-Alvarez, O., Kachatkou, A., Marchal, J., Willis, B., Sawhney, K., Tartoni, N. \& Van Silfhout, R. G. (2014). J. Instrum. 9, C12036.

Schildkamp, W. \& Pradervand, C. (1995). Rev. Sci. Instrum. 66, 19561959.

Seltman, H. (2017). Approximations for Mean and Variance of a Ratio. Department of Statistics at Carnegie Mellon University, USA. 
Shu, D., Kuzay, T. M., Fang, Y., Barraza, J. \& Cundiff, T. (1998). J. Synchrotron Rad. 5, 636-638.

Somogyi, A., Medjoubi, K., Baranton, G., Le Roux, V., Ribbens, M., Polack, F., Philippot, P. \& Samama, J.-P. (2015). J. Synchrotron Rad. 22, 1118-1129.
Spear, J. D. (2005). Rev. Sci. Instrum. 76, 076101.

Van Esch, P., Gahl, T. \& Guérard, B. (2004). Nucl. Instrum. Methods Phys. Res. A, 526, 493-500.

Zhou, T., Ding, W., Gaowei, M., De Geronimo, G., Bohon, J., Smedley, J. \& Muller, E. (2015). J. Synchrotron Rad. 22, 1396-1402. 